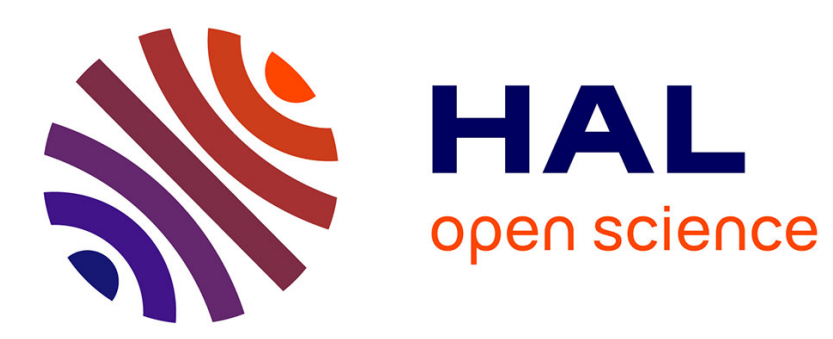

\title{
Catégorisations des migrantes par le religieux et agentivité autour de l'Islam
}

Simona Tersigni

\section{To cite this version:}

Simona Tersigni. Catégorisations des migrantes par le religieux et agentivité autour de l'Islam. Cahiers de la Méditerranée, 2009, 78, http://cdlm.revues.org/index4682.html. halshs-00623252

\section{HAL Id: halshs-00623252 \\ https://shs.hal.science/halshs-00623252}

Submitted on 15 Nov 2019

HAL is a multi-disciplinary open access archive for the deposit and dissemination of scientific research documents, whether they are published or not. The documents may come from teaching and research institutions in France or abroad, or from public or private research centers.
L'archive ouverte pluridisciplinaire $\mathbf{H A L}$, est destinée au dépôt et à la diffusion de documents scientifiques de niveau recherche, publiés ou non, émanant des établissements d'enseignement et de recherche français ou étrangers, des laboratoires publics ou privés. 


\section{CATEGORISATIONS DES MIGRANTES PAR LE RELIGIEUX ET AGENTIVITÉ AUTOUR DE L'ISLAM}

Simona Tersigni

Maître de conférences en sociologie des relations interethniques, Université de Rennes 2

UMR 6590 ESO (Unité de recherche " Espaces géographiques et sociétés »)

\section{Introduction}

Les catégorisations opérées sur les femmes et par les femmes en migration autour du religieux sont au centre de cette contribution. Alors que l'objet «femmes et immigration » en France s'est progressivement «ennobli»depuis la moitié des années Soixante-dix ${ }^{1}$, la religion des migrantes demeure encore une question négligée. Elle résulte des ambiguïtés d'analyses sur la féminisation de l'immigration qui se sont concentrées sur les « rejoignantes ». Dès cette époque cela a par ailleurs engendré une idéalisation du rôle nourricier et maternel des migrantes en France en même temps qu'une occultation des immigrées veuves et divorcées qui, déjà en 1982, sont quatre fois plus nombreuses que les hommes selon les données de l'INSEE².

Dans le cadre de l'immigration nord-africaine et post-coloniale, la religion a été étudiée du point de vue des migrants, notamment en relation avec la création de lieux de culte et du point de vue des «descendantes ». La rareté des travaux sur l'islam des migrantes ${ }^{3}$ renvoie à une double transformation dans les représentations, dont il importe de questionner le rôle des sciences sociales dans «la construction de leurs objets face à des catégorisations forgées pour asseoir des hiérarchies $»^{4}$. La première transformation relève du glissement de la minorité interne à l'étranger, puis à l'immigré et ensuite au musulman ${ }^{5}$. Allievi explique ce déplacement de catégorisations en fonction de la conjoncture (qu'il s'agisse des transformations de l'immigration en Europe ou de l'émergence de l'islam comme acteur global) et des évolutions à long terme liées à l'utilisation croissante des catégories interprétatives fondées sur la religion - l'islam représentant le cas extrême de cette utilisation. La seconde transformation renvoie à la récente visibilité que les « descendantes »

\footnotetext{
${ }^{1}$ Mirjana Morokvasic, "Femmes et genre dans l'étude des migrations : un regard rétrospectif», Cahiers du Cedref, «Femmes, genre, migrations et mondialisation : un état des problématiques », coordonné par Jules Falquet, Aude Rabaud, Jane Freedman, Francesca Scrinzi, Université Paris Diderot, avril 2008.

${ }^{2}$ Mirjana Morokvasic, op. cit., 2008, p. 42.

${ }^{3}$ Sossie Andezian, «Pratiques féminines de l'islam en France », Arch. Sc. Soc. des Rel., (1), 55, janvier-mars, 1983 ; Sossie Andezian, « Du 'religieux' dans les réseaux sociaux féminins », Arch. Sc. Soc. des Rel., (1), 68, juillet-septembre, 1989.

${ }^{4}$ Marco Martiniello et Patrick Simon, «La catégorisation et la classification comme enjeux de pouvoir. Rapports de domination et luttes autour de la représentation dans les sociétés post-migratoires », REMI, vol. 21, $\mathrm{n}^{\circ} 2,2005$.

${ }^{5}$ Stefano Allievi, «Comment l'immigrant est devenu le musulman. Débats publics sur l'Islam en Europe », REMI, vol. 21, $\mathrm{n}^{\circ} 2,2005$.
} 
musulmanes ont acquise sur les hommes musulmans ${ }^{6}$. Cet autre déplacement s'est opéré dans une conjoncture scientifique précise dans laquelle les descendantes de migrant(e)s ont cessé d'évoquer la religion pour légitimer leur engagement dans la mise en œuvre collective des revendications publiques qui s'appuient sur l'islam. Comme le souligne Amiraux, au moment où les sciences sociales analysent le rapport que les musulmanes entretiennent avec la foi en termes d'identification, de pratiques et d'engagement, ces jeunes filles font davantage référence aux droits de la personne pour porter leurs revendications. En effet, l'hypervisibilité contemporaine de la religion des « descendantes » est le produit d'une «succession d'invisibilisations emboîtées ${ }^{7}$ de la religion ou de la non religion des migrantes dans le cadre de la sociologie française des migrations. Celle-ci, initialement marquée par l'absence de travaux sur les pratiques religieuses en migration, notamment du côté des femmes (1960 1985), finit par construire le religieux en tant qu'objet légitime en évacuant le rôle des migrantes. Il faudra attendre le début des années 2000 pour que des sociologues des migrations commencent à aborder frontalement les dissymétries hommes/femmes autour du religieux et les tactiques religieuses spatialisées du point de vue du système sexe-genre ${ }^{8}$.

En France le retard dans le traitement sociologique des religions en migration n'a pas empêché le recours aux religieux comme mode de catégorisation des populations migrantes. Néanmoins, du point de vue de l'analyse, il importe de penser les migrantes comme des sujets collectifs minorisés mais non complètement agis. Insister sur le caractère dialectique du processus de catégorisation comme le font Bail et Lamont ${ }^{9}$ revient à souligner que «le minoritaire est aussi un acteur central dans la catégorisation, la classification et partant la construction identitaire $\gg{ }^{10}$ faites d'oppressions imbriquées. L'architecture de ce texte les traite à partir de l'agentivité des migrantes par le religieux (premier paragraphe) pour ensuite appréhender de manière plus fine la division sexuelle du travail religieux et les marges de manœuvre des migrantes (deuxième paragraphe). Les trois derniers paragraphes complètent cette analyse autour du lien entre agentivité féminine et mise sous tutelle masculine de la

\footnotetext{
${ }^{6}$ Valérie Amiraux, «Discours voilés sur les musulmanes en Europe. Comment les musulmans sont-ils devenus des musulmanes », Social Compass, vol. 1, n 50, 2003.

${ }^{7}$ Simona Tersigni, « Jalons pour une lecture imbriquée du genre et du religieux dans le champ des migrations et des relations interethniques en France », Cahiers du Cedref, « Femmes, genre, migrations et mondialisation : un état des problématiques », coordonné par Jules Falquet, Aude Rabaud, Jane Freedman, Francesca Scrinzi, Université Paris Diderot, avril 2008.

${ }^{8}$ Simona Tersigni, Les frontières du partage : relations interethniques et approches de l'islam. Discours et pratiques de migrantes d'Afrique du Nord et de leurs filles, thèse, sociologie, Paris 7, 2003, 407 p. ; Mahamet Timera, "Islam et renégociation des rapports de genre en contexte migratoire », in Isabel Taboada-Leonetti, (ed.), Les femmes et l'islam : entre modernité et intégrisme, Paris, L'Harmattan, 2004 : 155-161 ; Fatima Aït Ben Lmadani, La vieillesse illégitime ? Migrantes marocaines en quête de reconnaissance sociale, thèse, sociologie, Paris 7, 2007.

${ }^{9}$ Christopher Bail et Michèle Lamont, «Sur les frontières de la reconnaissance. Les catégories internes et externes de l'identité collective », REMI, vol. 21, n 2, 2005.

${ }^{10}$ Marco Martiniello et Patrick Simon, op. cit., 2005, p. 6.
} 
transmission religieuse en migration (troisième paragraphe); entre transmission religieuse « remontante » opérée par les « descendant(e)s » et invention de la tradition et, enfin, entre transmission maternelle de l'islam et mise en concurrence avec les leaders religieux.

\section{Oppressions imbriquées et agentivité des migrantes par le religieux}

De nombreuses spécialistes de l'histoire des femmes estiment que le champ d'action des femmes pour la transmission de la foi et des pratiques religieuses s'est élargi au détriment des hommes, précisément au moment où les liens entre le religieux et le politique sont rompus. En France, en matière des droits des femmes plusieurs laïcités se sont affrontées sur les modalités de la régulation du politique et du religieux, avec des incidences sociales sur les équilibres de genre ${ }^{11}$. Pourtant cette thèse n'a pas spécialement orienté les recherches du champ français des migrations et des relations interethniques. Sa progressive mise en place ne s'est pas immédiatement accompagnée de travaux centrés sur le rapport que les migrantes entretiennent avec l'Au-delà ni sur les contraintes auxquelles elles seraient soumises vis-à-vis de la société majoritaire, de leur « groupe » et des migrants partageant leur même foi.

Par l'approche du travail salarié, de la participation politique et sociale, de la citoyenneté des migrantes, de nombreuses recherches ont déjà contribué à questionner l'image de passivité qui est accolée aux migrantes. Toutefois, il est manifeste qu'une analyse des femmes et des hommes en migration dans une perspective de genre peut être élargie au religieux. De plus, quand les migrant(e)s sont croyant(e)s et/ou soumis(es) à des catégorisations prenant appuis sur la religion, on peut s'interroger sur les multiples manières dont leurs descendantes arrivent à assumer ou non les liens tissés avec les parents. Acceptentelles ou non leur héritage symbolique et matériel en matière de religion? En voulant le reproduire, comment le réinvestissent-elles dans un contexte d'ethnicisation des rapports sociaux qui les renvoie à leurs « origines » nationales, culturelles, religieuses ? Cela implique une rupture avec une lecture unique d'inéluctable oppression dans le lien entre femmes et religion en même temps qu'avec l'idée d'un rapport homogène des migrantes à la religion. Si des migrantes ou des descendantes de migrant(e)s croyant(e)s ou assignées à des catégorisations de type religieux entretiennent éventuellement un rapport spécifique à la religion «c'est en raison de la place qu'elles occupent, en tant que femmes, dans les rapports

\footnotetext{
${ }^{11}$ Florence Rochefort, "Laïcité et droits des femmes. Quelques jalons pour une réflexion historique », Arch. de Phil. de droit, vol. 48, 2004.
} 
sociaux. Et c'est cette condition sociale qui commande la place qu'elles occupent dans le champ religieux $»^{12}$.

La thèse du strict lien entre laïcité et droits des femmes a été élaborée dans un contexte européen sécularisé où la religion continue d'alimenter l'imaginaire qui régit la perception de soi et de l'autre. Pour reprendre une formulation chère à Monder Kilani ${ }^{13}$, il y a eu «persistance du facteur religieux en tant que catégorie de traduction des différences culturelles ». Cet imaginaire religieux n'est pas forcément celui d'une adhésion à un contenu religieux ou à une confession, mais celui d'une identification culturelle et historique. Dans ce cadre de représentations, ceux et celles qui figurent comme allogènes peuvent être perçu(e)s en termes religieux, alors même qu'ils ne s'identifient pas tous et toutes par et à travers cette dimension. Des sujets, parce que d'ascendance allogène (Europe du Sud, Europe de l'Est, Maghreb, Afrique occidentale, Proche Orient) se sont vus préalablement attribuer des catégorisations sur un fond religieux. Ces labellisations ne les concernaient pas nécessairement et ils ont dû néanmoins prendre position vis-à-vis de celles-ci par des formes d'agentivité qui ne sont pas automatiquement «synonyme de liberté et d'égalité pleines et entières $»^{14}$. Travailler sur la division sexuelle du travail religieux requiert alors de ne pas sous-estimer les oppressions imbriquées qui pèsent sur les migrantes et sur leurs descendantes, ni d'oublier l'hétérogénéité « de classe, d'âge, de génération, de nationalité, d'imputation ethnique, d'assignation « raciale », de statut légal, de position dans l'institution familiale, ou encore de pratiques sociales et politiques $»^{15}$.

\section{Division sexuelle du travail religieux et marges de manœuvre féminins}

En tant que volonté d'action et positionnement de sujets politiques individuels et collectifs, l'agentivité n'exclut pas d'étudier la division sexuelle du travail religieux en migration à partir de la mobilisation de ressources religieuses (et plus en général culturelles) acquises dans la famille au cours de la socialisation. Cette division sexuelle du travail religieux minoritaire peut se reproduire même dans l'espace public, au cours d'interactions ponctuelles inattendues, qui ne relèvent ni de comportements réflexifs assumés tels que le port

\footnotetext{
${ }^{12}$ Danièle Hervieu-Léger, Introduction, in « Oppression des femmes et religion », Colloque de l'association française de sociologie religieuse, Travaux et documents, VIII, 1983.

${ }^{13}$ Monder Kilani, «Il faut déconfessionnaliser la laïcité. Le religieux imprègne encore les imaginaires », Journal des anthropologues, $\mathrm{n}^{\circ}$ 100-101, 2005.

${ }^{14}$ Jules Falquet et Aude Rabaud, Introduction, Cahiers du Cedref, « Femmes, genre, migrations et mondialisation : un état des problématiques », coordonné par Jules Falquet, Aude Rabaud, Jane Freedman, Francesca Scrinzi, Université Paris Diderot, avril 2008.

15 Jules Falquet et Aude Rabaud, op. cit., p. 25.
} 
habituel du foulard ${ }^{16}$ ni de la sectorisation des activités selon les sexes, comme il arrive dans les contextes associatifs militants ${ }^{17}$. De plus, les imbrications entre histoire familiale et engagement religieux rencontrées dans le travail d'enquête sont très souvent mentionnées, sans qu'elles ne soient pas vraiment conceptualisées par les chercheur(e)s qui s'y intéressent. En revanche, nous nous sommes concentrée sur la manière dont les mères orientent l'incorporation de pratiques, y compris quand les acteurs sociaux s'en éloignent ou affirment, du moins, s'en être coupés.

Bien qu'il ne concerne pas directement des migrants et leurs descendants, le brillant article de Julien Fretel ${ }^{18}$ sur les militants catholiques de l'UDF souligne le rôle d'une «pédagogie parentale sans cesse continuée » ayant influencé la future socialisation politique de ses interlocuteurs. Axée sur le capital religieux militant, la perspective de Fretel permet de mieux approfondir le rôle des mères dans une socialisation religieuse genrée en migration. Toutefois, si Julien Fretel se limite à emprunter la notion de « socialisation au désintérêt » de Jacques Maître ${ }^{19}$, il nous semble que les multiples formes de transmission et de non transmission féminine qui organisent la vie sociale ne peuvent pas être dissociées des constructions idéologiques, du sentiment de leur légitimité et illégitimité ni de l'ordre social qui en résulte ${ }^{20}$. C'est pourquoi les différentes injonctions de transmission faites aux migrantes par leurs hommes et les pratiques de contrôle qui s’y réfèrent, sont au cœur du système sexe-genre, faisant glisser l'analyse du domaine strictement migratoire vers les relations interethniques. Il s'agit alors de s'interroger sur la transmission du côté de celles qui la pratiquent (les mères) et non seulement de ceux et celles qui la reçoivent, tout en saisissant le lien avec les logiques de reprise en main institutionnelle (et masculine) de l'autonomie religieuse grignotée par les femmes. Car la contribution complexe des migrantes à une transmission religieuse s'analyse à la fois comme inscription symbolique dans une « lignée croyante $»-i . e$. en tant qu'introduction pratique à une expérience religieuse à caractère émotionnel - , mais également en tant qu'acquisition d'un savoir spécifique en relation ou non avec des institutions religieuses ${ }^{21}$.

\footnotetext{
${ }^{16}$ Simona Tersigni, «La pratique du hijâb en France. Prescription, transmission horizontale et dissidence », in F. Lorcerie, ed., La Politisation du voile. L'affaire en France et son écho à l'étranger. Paris, L'Harmattan, 2005.

${ }_{17}$ Marie Lejeune et Simona Tersigni, «L'école publique comme espace de revendication individuelle et collective en France : l'autochtonie par l'islam », Journal des Anthropologues, n¹00-101, 2005.

${ }^{18}$ Julien Fretel, «Quand les catholiques vont au parti. De la constitution d'une illusion paradoxale et du passage à l'acte chez les 'militants' de l’UDF », ARSS, n¹55, décembre, 2004.

${ }^{19}$ Jacques Maîtres, Anorexies religieuses, anorexie mentale. Essai de psychanalyse sociohistorique, Paris, Cerf, 2000.

${ }^{20}$ Gérard Lenclud, "Transmission», in Pierre Bonte et Michel Izard (eds.), Dictionnaire de l'ethnologie et de l'anthropologie, Paris, PUF, 1991.

${ }^{21}$ Danièle Hervieu-Léger, La religion pour mémoire, Paris, Ed. du Cerf, 1993.
} 


\section{Agentivité et mise sous tutelle masculine de la transmission religieuse en migration}

Les enjeux de l'ensemble des activités qui participent à la reproduction des êtres humains sur le plan du travail domestique comme des processus liés à la reproduction corporelle (soins médicaux, socialisation aux normes culturelles, enseignement...) concernent l'ensemble des sujets regroupés sous les mêmes catégorisations de Nord-Af', Arabes, Beurs, Rebeux, musulmans. L'on assiste tout de moins à une focalisation sur les devoirs des femmes à savoir faire (préparation des repas, soins aux enfants en bas âge, entretien matériel du foyer) et à savoir transmettre. Dans le cadre de nos recherches doctorales ${ }^{22}$, en octobre 2000 nous nous sommes rendues à la cité des Sciences et de l'industrie (la Villette) de Paris, où des chercheuses du Centre National de la Recherche Scientifique (CNRS) proposent un débat après la projection d'un documentaire tourné au moment du Ramadan et de la fête du mouton. A cette occasion, l'on voit des mères qui apprennent à leurs filles à tourner les galettes de pain sur la plaque chaude, à préparer d'autres plats festifs et, surtout, à partager les nourritures. Après l'intervention d'une jeune ethnologue, au cours du débat, une femme qui se dit kabyle raconte comment, malgré son fort désir de se cultiver à l'école durant son adolescence, elle était obligée par sa mère de s'occuper de ses frères et sœurs, de la maison, bref d'apprendre à devenir une épouse « sage ». «J'étais une jeune femme révoltée dans les années Soixante-dix, qui a fait des études même si ce n'était pas la priorité de mes parents ». Mais sa mère ne lui a pas vraiment transmis la langue kabyle, qu'elle a en revanche apprise en autodidacte et puis transmise à sa fille, au lieu de la cuisine («elle l'apprendra un jour, si elle veut »)... Assise à côté d'elle, sa fille confirme publiquement : « Ce qui est important pour ma génération est la façon dont on nous transmet », si avant l'enjeu était l'acceptation d'une jeune femme par sa belle-famille, aujourd'hui, dit-elle, «je n'ai pas de compte à rendre, nous cuisinerons autrement!». Puis mère et fille sont d'accord sur le fait que ce savoir-faire se transmet indépendamment de toute imposition, par le regard et même lors de coups de fils en urgence pour avoir une recette de maman... Dans la salle une femme-relais évoque ensuite la mise en place - et le succès chez des adolescentes - d'ateliers de cuisine dans un certain nombre de quartiers d'habitat social, confirmant que la cuisine traditionnelle n'est pas complètement rejetée même si elle est apprise dans des cadres qui ne sont pas uniquement ceux de la maison, avec les femmes de la famille... Suit l'intervention d'un monsieur dans la salle : «Quand les enfants ont la chance d'avoir une mère qui leur transmet ce qu'il faut savoir

\footnotetext{
${ }^{22}$ Simona Tersigni, op. cit., 2003.
} 
dans la vie... ». Il s'insurge ainsi contre ces femmes qui s'abstiennent de ce qu'il considère comme étant leur devoir principal dans un contexte migratoire, soit la sauvegarde des valeurs domestiques et la transmission de l'islam. Il humilie à la fois cette mère et sa fille tout en soulignant le rôle secondaire des hommes, y compris lors des fêtes, dans un travail qui relève pour lui de la spécificité féminine.

En effet, la focalisation sur les femmes n'a pas de contrepartie pour ce qui est des savoir-faire rituels masculins, en matière de sacrifice en particulier. Certains hommes s'arrogent alors le droit d'indiquer publiquement aux femmes elles-mêmes en quoi consisteraient leurs tâches domestiques et quelles pratiques devraient faire l'objet de transmission, comme nous l'avons remarqué lors de plusieurs rencontres et débats publics qui à l'origine ne portaient pas directement sur la transmission. Les femmes «musulmanes » ont des responsabilités conséquentes dans les identifications culturelles de leur propre " groupe » et dans une mise en image connexe vis-à-vis des acteurs majoritaires. Compte tenu des enjeux attachés à cette transmission pour les pratiques minoritaires, les responsabilités des hommes paraissent moindres : ils ne sont pas chargés du même poids pour leur "groupe », ce qui aboutit parfois aux crispations de ceux qui se posent en tant que juges normatifs de l'émancipation féminine.

\section{Transmission religieuse « remontante » et invention de la tradition en migration}

Dans ses recherches menées auprès des familles sépharades de Sarcelles, Annie Benveniste $^{23}$ montre un paradoxe qui est tout aussi valable pour les familles d'ascendance nord-africaine aujourd'hui. Les mères migrantes continuent à assurer la transmission des valeurs domestiques et à en fonder le sens. Toutefois, si les mères sont les garantes des traditions culturelles et religieuses à l'intérieur du foyer - en assurant l'éducation des enfants en bas âge et le maintien des pratiques alimentaires - elles sont souvent dépassées par leurs enfants qui prétendent les connaître mieux qu'elles. Ainsi, ces « descendant(e)s » tentent d'imposer à leurs mères des stratégies identitaires multiples et d'autres codes normatifs empruntés à des formes d'invention de la tradition ${ }^{24}$ : il suffit de penser à la consommation de viande halal ou au régime de vie lié au port du foulard.

Les rôles d'autorité entre générations sont parfois inversés par une transmission religieuse «remontante» chez les Sépharades comme dans les familles musulmanes. La perception essentialiste de l'islam qui en a fait une religion incompatible avec les principes

\footnotetext{
${ }^{23}$ Annie Benveniste, Figures politiques de l'identité juive à Sarcelles, Paris, L'Harmattan, 2002.

${ }^{24}$ Eric Hobsbawm et Terence Ranger (eds.), The Invention of Tradition, Cambridge, Cambridge University Press, 1983 ; Déjan Dimitrijevic et Eric Hobsbawn, Fabrication de traditions. Invention de modernité, Paris, Editions MSH, 2004.
} 
républicains et la modernité ainsi qu'un danger pour la cohésion sociale de la France a été en effet plaquée sur leurs descendant(e)s. Considéré(e)s comme nécessairement bénéficiaires d'une socialisation religieuse, ils/elles sont perçu(e)s comme ne pouvant qu'adhérer au credo musulman, lequel devrait nécessairement primer sur les autres composantes de l'héritage culturel $^{25}$. L'on pouvait se douter du fait que cette passivité présumée face à la transmission n'en est pas une. Parmi les descendant(e)s de migrants qui adhèrent aux formes de fidélisation islamique, indépendamment d'une adhésion savante ou non à l'islam, l'affiliation au « groupe » (les musulmans de France/les Arabes-Rebeux-Nord-Africains/nombre restreint de militants...) s'effectue par l'autonomisation de leurs propres décisions basées sur l'islam auprès des parents et par la distanciation du processus maternel de filiation.

\section{Transmission maternelle de l'islam et mise en concurrence avec les leaders religieux}

Soulimane $^{26}$, le fils d'une de nos interlocutrices rencontré pendant nos recherches doctorales, considère qu'à l'occasion de l'Aïd el-kebir il ne faudrait surtout pas susciter un sentiment de dégoût de la part des Français à l'égard des musulmans par le sacrifice du mouton... Il déclare : "Nous ne sommes toujours pas chez nous en France » pour ensuite mettre l'accent sur les complications d'ordre «hygiénique » liées au sacrifice. D’ailleurs, dégoûté par l'odeur de la viande du mouton sacrifié lors de l'Aïd, il refuse d'en manger depuis cinq ans. Sa vision de l'islam est - sans qu'il le sache - calquée sur les positions institutionnelles que les représentants de la mosquée de Paris ont défendues à la fin des années 1980 - positions qui s'accommodent de la vision majoritaire « autochtone ${ }^{27}$. Aussi, la pudeur des jeunes filles qui «prennent le foulard» s'est construite sous le regard normatif quotidien des mères. Les effets de l'inculcation de cette pudeur ne manquent pas d'étonner ces mères mêmes, et certaines sont parfois frappées par les attitudes qu'elles jugent excessives de la part de leurs filles ${ }^{28}$. Par exemple, contrairement à leurs mères, ces jeunes filles ont

\footnotetext{
${ }^{25}$ Nathalie Kakpo, «L'islam pour renégocier sa place dans les institutions publiques : l'exemple des animateurs de centres sociaux ", in Jean-Paum Payet, Frédérique Giuliani et Denis Laforgue (eds.), La voix des acteurs faibles. De l'indignité à la reconnaissance, Rennes, Presses Universitaires de Rennes, coll. Le sens social.

${ }^{26}$ Adolescent âgé de 13 ans, né à Nanterre de mère immigrée d'Algérie et résidant à Nanterre depuis l'âge de 16 ans.

${ }^{27}$ La vision de Soulimane rappelle la position de Abbas Cheikh, défunt recteur de l'Institut musulman de Paris. En 1987 il a publiquement soutenu que le sacrifice du mouton attire un jugement négatif sur l'islam et n'a plus de place en France. En proposant aux fidèles de remplacer le sacrifice du mouton par "l'égorgement domestique et discret d'un poulet", ce représentant d'un islam républicain, demandait aux musulmans de «garder la part intime, spirituelle et se détacher de la part manifeste " de l'islam, de choisir entre ce qui pouvait être transmis dans le cadre de la France et ce qui risquait de « choquer» les non musulmans. L'invitation adressée par ce recteur à des hommes musulmans constitue une sorte de double injonction liée au regard de la société française. S'abstenir du sacrifice du mouton implique ici deux sortes de réductionnismes pour les hommes musulmans. D'une part, il s'agit de se «féminiser » par le biais du sacrifice d'un poulet souvent associé à des rituels populaires féminins d'ordre magico-religieux. D'autre part, cela implique la perte d'un savoirfaire associé à un rituel primordial tel que la fête du mouton vis-à-vis de l'islam lui-même, autant qu'en relation avec le statut de père et de la responsabilité de son pouvoir découlant directement du divin.

${ }^{28}$ Simona Tersigni, « La pratique du hijâb..., op. cit., 2005.
} 
tendance à ne pas ôter leur hijâb quand elles se retrouvent entre elles, même dans leur domicile...

En effet, maints chercheur(e) $\mathrm{s}^{29}$ ont mis l'accent sur le fait que dans la transmission de l'islam à leurs enfants, les parents migrants - dont l'expérience d'un islam intellectualisé est rare -, se sont vus concurrencer par les activités associatives de leaders religieux dans le cadre des besoins d'animation socio-culturelles et de soutien scolaire des quartiers ségrégués. Toutefois il a très rarement été souligné combien la distanciation s'effectue, du moins en termes d'énonciation, par le branchement sur des positions institutionnelles ou contestataires conduisant à faire aux parents migrants les mêmes reproches que ces recteurs ou leaders religieux pourraient leur adresser. Dans tous les cas, les positionnements des enfants par rapport à l'islam peuvent engendrer des réaménagements de la religiosité des parents.

En particulier, certaines mères ont appris à se démarquer des discours et des pratiques qu'elles considèrent comme étant islamistes et à incorporer dans la transmission de la religion des contre-outils et des contre-témoignages. Fêter l'anniversaire du Prophète (Mawlid) dans les locaux associatifs d'un club de prévention de Nanterre effectuant aussi du soutien scolaire marque une pratique de distinction vis-à-vis des «mecs en gandoura, qui sont un peu barbus, avec leurs Nike dernier cri, et qui font du 'soutien scolaire islamique’ ». De plus, pour les mères musulmanes originaires du Sud de la Tunisie et de l'Oranais en Algérie notamment, il s'agit d'un véritable calcul religieux. "Marquer le coup le jour du Mawlid» leur permet de contrer les influences directes et indirectes des leaders religieux en rappelant la place des mères musulmanes qui accouchent et font des futurs musulmans, "peu importe si cette fête n'est pas dans la liste des fêtes musulmanes à La Mecque, car on l'a toujours fêtée en famille». Cette fête est très controversée car elle n'est pas officiellement reconnue par tous les musulmans et souvent reléguée dans la coutume... Le Mawlid, en tant que fête honorant la place des mères musulmanes et relevant de traditions populaires, ne se trouve pas au centre des revendications collectives. Il s'agit d'une fête minoritaire parmi les minoritaires qui n'est pas inscrite dans l' "intérêt collectif » défini pas les militants associatifs musulmans comme par les représentants institutionnels de l'islam de France. Le Mawlid rend localement visibles

\footnotetext{
${ }^{29}$ Leila Babès « Recompositions identitaires dans l'islam en France. La culture réinventée », Arch. Sc. Soc. des Rel, n 92 , 1995 ; Chantal Saint-Blancat, «Une diaspora musulmane en Europe? », Arch. des sciences soc. des religions, $n^{\circ}$ 92, 1995, Nadine Weibel, «Islamité, Egalité et Complémentarité : vers une nouvelle approche de l'identité féminine », Arch. Sc. Soc. des Rel., $\mathrm{n}^{\circ}$ 95, 1996, Farhad Khosrokhavar, L'islam des jeunes, Paris, Flammarion, 1997, Nikola Tietze, Jeunes musulmans de France et d'Allemagne. Les constructions subjectives de l'identité, Paris, L'Harmattan, 2003, Simona Tersigni, Les frontières du partage..., op. cit., Nancy Venel, Musulmans et citoyens, Paris, PUF, 2004, Amel Boubekeur, "L'islamisme comme tradition. Fatigue militante et désengagement islamiste en Occident », in Samir Amghar (ed.), Islamismes d'Occident. Etat des lieux et perspectives, Paris, Editions Lignes de Repères, 2006.
} 
des migrantes et légitime une approche non savante de la religion, sans pour autant vouloir en faire des « citoyennes musulmanes ».

\section{Conclusion}

L'enjeu des migrantes musulmanes se situe par rapport à la distanciation effectuée par les enfants à l'égard de la transmission maternelle. Celle-ci s'inscrit en effet dans les rapports sociaux de sexe. A l'origine des positionnements de ces jeunes musulmans se trouvent des figures masculines - qu'elles soient antagonistes comme dans le cas des leaders charismatiques ou institutionnels comme dans le cas du recteur de l'Institut musulman de la mosquée de Paris. L'islam de ces migrantes a priori n'est ni orthodoxe (au sens où ce qualificatif associe une pensée cohérente d'éléments doctrinaux et la reconnaissance d'un pouvoir y afférent) en soi, ni anti-islamiste en soi, ni populaire et magico-religieux en soi. C'est donc en se positionnant vis-à-vis des agissements et des discours de ces figures contemporaines de l'islam de France, face aux injonctions masculines et à la société majoritaire «autochtone» que les migrantes redéfinissent leur islam. Elles le font collectivement (lors d'échanges au marché comme pendant des activités associatives dans leur quartier de résidence, ce qui n'exclut pas non plus les conflits) et individuellement dans la quotidienneté d'une transmission qui a vocation à devenir de plus en plus consciente de l'illusion de l'automatisme de la répétition. 